\title{
The importance of contextualisation in giving a diagnosis of genital chlamydial infection: findings from a qualitative study
}

Hilary Piercy

\begin{abstract}
Objective To explore the impact of diagnostic communication upon the way in which people receive and respond to a diagnosis of chlamydial infection.

Methods A qualitative study examining the responses of individuals to a diagnosis of chlamydial infection. The study was conducted in a genitourinary medicine clinic and a family planning clinic in the Midlands region of the UK. The sample size was 50 and included both males and females. Data collection was by means of unstructured interviews, which were audio-taped and fully transcribed. The principles of grounded theory were followed in the sampling, analysis and exploration of the literature.
\end{abstract}

Results A diagnosis of chlamydial infection was commonly unexpected and associated with negative reactions, which derived from the social construction of sexually transmitted infections as evidence of breaching the moral code. The way in which the health professional communicated the diagnosis contributed to the patient response, either negatively by reinforcing feelings of self-recrimination or positively by the provision of key information that appeared to be helpful in modifying that response.

Conclusion Sensitive management and the provision of contextualised information serve an important function for those diagnosed with chlamydial infection.

J Fam Plann Reprod Health Care 2006; 32(4): 227-230

(Accepted 12 February 2006)

\section{Key message points}

- The diagnosis of chlamydial infection is a potential cause of emotional upset and distress that is associated with feelings of bodily contamination.

- At the point of diagnosis the sensitivity of the information heightens the significance of what is said and how it is said.

- Insensitive management by health professionals may impact negatively upon the response to diagnosis.

- Contextualisation of diagnosis by the provision of key information appears to be helpful in modifying the response to diagnosis.

School of Nursing and Midwifery, University of Sheffield Sheffield, UK

Hilary Piercy, RGN, PhD, Lecturer

Correspondence to: Dr Hilary Piercy, School of Nursing and Midwifery, University of Sheffield, Bartolome House, Winter Street, Sheffield S3 7ND. E-mail: h.piercy@sheffield.ac.uk

\section{Introduction}

Chlamydia is the most commonly diagnosed sexually transmitted infection (STI) in England ${ }^{1}$ with an estimated population prevalence rate of $5.0 \%$ in those aged under 20 years $^{2}$ and considerably higher rates in selected populations. ${ }^{3}$ Efforts to reduce the rate of this infection, which has considerable implications for sexual and reproductive health, have increased over the past few years. The national chlamydia screening programme, currently being implemented across the country and expected to achieve national coverage by 2007 , represents an important part of this effort. This opportunistic programme targets those aged under 25 years and operates predominantly through primary care facilities such as general practice and sexual health clinics. Consequently, primary health care professionals are becoming increasingly involved in the management of this infection.

There is an increasing move towards detection of chlamydia using samples such as urine and self-taken vaginal swabs. These increase the acceptability and ease of testing for patient and professional alike 4 and are likely to contribute to the routinisation of testing for chlamydia. However, diagnosis often has considerable impact upon the individual, being associated with notions of dirt, pollution and contamination. 5,6

Stigma has been categorised as felt and enacted stigma. ${ }^{7}$ It is specifically felt stigma, whereby the person experiences a fear of disclosure and an expectation of social sanctioning, which has been acknowledged in relation to STIS $^{8,9}$ and recognised as contributing to people's reluctance to access treatment. ${ }^{10-12}$ The point of diagnosis is important as the time at which such feelings may be realised and as an opportunity when they may be reinforced or challenged. This paper reports on findings from a qualitative study that explored the experiences of those treated for chlamydia, in particular those factors which influence an individual's response to diagnosis.

\section{Methods}

In-depth unstructured interviews were conducted with 50 individuals (40 females aged 16-29 and 10 males aged 18-33 years) who had been diagnosed with and treated for chlamydial infection. The mean age was 20 years and the median age was 17 years. The majority of the interviews ( $n$ $=44$ ) were conducted in a genitourinary medicine (GUM) clinic based within a medium-sized district general hospital serving a semi-rural population with $1 \%$ ethnic minority in the Midlands region of the UK. Recruitment was opportunistic, and managed by the medical and nursing staff. The researcher was based in the unit for 1 day per week. Patients attending the clinic on that day in relation to chlamydial infection, either for diagnosis and treatment or follow-up (which was clinic policy at the time), were identified by staff and invited to participate in the study. They were offered the opportunity to be interviewed at that time or at a later date. All but one agreed to be interviewed at the time.

The second study site was the town centre family planning clinic (FPC) serving the same population. Those attending the clinic whose records indicated that they had 
had chlamydial infection within the last 12 months were invited to participate. The purpose of this approach was to gain a longer-term perspective from those individuals for whom the infection was a less recent event and to provide a means by which to access those who had not attended the GUM clinic. Recruitment difficulties limited the respondents to six females, all but one of whom had attended the GUM clinic in relation to their infection episode.

Exclusion criteria were those under 16 years of age for reasons of consent and those whose diagnosis had occurred prior to termination of pregnancy in consideration of the additional emotional elements associated with such a procedure. Interviews lasted between 10 minutes and 1 hour and took place in a consulting room in the clinic at the end of the patient's visit.

All interviews were conducted by the researcher and tape-recorded with the permission of the respondents. The interviews were fully transcribed. Pseudonyms and ages are included with the data extracts in this paper. The study had ethical approval from the local research ethics committee.

\section{Data analysis}

The data were analysed in accordance with the principles of grounded theory ${ }^{13-15}$ using a coding and categorisation process. Initially, open codes were assigned to the data that reflected what had been said and what was going on. These open codes were then categorised and clustered. This process resulted in the development of core categories that were grounded in both the data and relevant theoretical concepts.

\section{Results}

\section{Impact of diagnosis}

For the women in this study, diagnosis of infection had occurred either in primary care $(n=25)$ or in the GUM clinic $(n=15)$. For the men, testing and diagnosis had occurred solely in the GUM clinic. Most of the respondents had presented with symptoms that resulted in them being tested for infection. In the majority of cases the diagnosis of chlamydial infection was unexpected and unanticipated even though most had known that one of the primary purposes of the testing to which they had submitted themselves was the detection of STIs. Diagnosis commonly produced a general feeling of bodily discomfort, which was experienced and expressed as a strong sense of contamination and a challenge to their sense of self.

"I felt dirty, I felt dirty, I just felt really dirty and that it shouldn't have happened to me." [Paula, 19]

"I thought I was dirty, I thought, I can't be dirty." [Steve, $18]$

The stigmatising effect of STIs is founded upon the social construction of these infections, which over the course of history have been associated with disreputable and immoral people and activities, their presence being taken as indicative of a breach of the moral code. This viewpoint may seem to reside in the historical legacy of such an infection and therefore have little currency in the present time, however the data indicated that it remains a prevalent societal view, resistant to the passage of time and the impact of public health efforts. Certain kinds of amoral behaviours were considered to predispose to infection and these behaviours were particularly associated with certain kinds of people.

"I knew about them, but I don't put myself in that group, do you know what I mean, like you put them in a group, like people that get diseases .... I didn't consider myself that type of person, I thought you had to be tarting about all the time to get owt like that." [Judy, 28]

The strength of response was variable and was not inevitable. Some, such as Sue (17) rejected the notion of dirtiness, considering that she had simply "slept with the wrong person", whilst others such as Sheila (29) were primarily relieved that the infection had been detected. Notwithstanding this, expressions of pollution or stigma arose spontaneously in more than two-thirds of the interviews. Many of those respondents described expressions of upset and distress, several of them being reduced to tears on discovering the diagnosis.

\section{Communicating the diagnosis}

The impact of diagnosis was tempered by the way in which it was communicated. Several respondents, all of whom had been diagnosed in primary care, described how they considered the situation to have been insensitively managed by the health professional. They perceived that the service provider morally condemned their sexual practices, which produced and intensified feelings of bodily pollution. This perception resulted from the way that the initial diagnosis, either in general or specific terms, was presented. For several of the respondents, the effect of this was a deeply distressing experience.

"I was not expecting that at all, not at all, and I just think that she [the doctor] could have erm, said it a bit nicer to me instead of making me feel so cheap and dirty, because she made me feel that way." [Jane, 26]

"Everytime I just felt so dirty, like when they [the primary care providers] was talking to me, like really really bad. I don't know, I just felt like a slapper, and I'd not done anything wrong really and that's just the impression that they were looking over me, wagging a finger at me sort of thing and I'd not done anything wrong." [Sian, 20]

The way in which the diagnosis was presented was important. If there was no accompanying discussion, or if there was an attempt to locate the infection in the behaviour either of the patient or her partner, then it had the effect of communicating not only the diagnosis but also the social construction of it, and the emotional response was amplified. Although the health professional may not have intended to convey a moral context when they were communicating the diagnosis, the sensitivity of the information heightened the significance of what was said and what was implied. In those who described extreme distress, the primary perception was of a diagnosis delivered in a detached and matter-of-fact way.

"She came out basically oh yes, it looks very sore inside, well she said you've either had a miscarriage or it's chlamydia. Well, I've never, the way that I felt when she told me that was unbelievable. I thought she was very callous in the way she said it." [Alison, 26]

"When she said oh, I think it's a sexually transmitted disease, if you've got any pain in your ovaries it's a sexually transmitted disease and I was shocked, I cried." [Jane, 26]

It was the starkness of the information that was considered to be so traumatic, and the lack of explanation or opportunity for discussion or clarification that contributed to the emotional effect.

"She said, oh, it might not be, I'm only surmising, I thought oh thanks, she didn't offer me any sort of explanation, she 
didn't tell me about chlamydia, I didn't know anything about it." [Alison, 26]

Alternatively, any comments that suggested a location for the source of the infection, by making inferences on the basis of the information supplied, were also distressing to the respondent because they potentially impacted upon their relationship status.

"They said that I'd got chlamydia and basically, have you been with anyone else, and I told them what I told you [a stable relationship for 6 years] and they said well, it can't be from you then, it must be from someone else. Is he seeing someone else?" [Sian, 20]

It is difficult to imagine that those health professionals who made such statements could have had any concept of the effect that their dismissive attitude or their conjectures had upon the recipients of such information. However, the data underline how important the communication is at this point and the imperative of considering the impact of what is said upon someone in such a sensitive state.

\section{Modifying the impact of diagnosis}

When health professionals provided explanations, these constituted the means by which to challenge and therefore effectively undermine the impact of a chlamydial diagnosis. Certain key pieces of information appeared to have particular significance in this respect because they represented a tangible way of undermining the feeling of bodily pollution that accompanied diagnosis. By presenting the diagnosis in a contextualised way, many of the initial concerns and anxieties could be allayed. The importance of such information lay in the fact that it addressed the very common questions asked at the time of diagnosis and it provided the means through which to challenge the preconceptions and the social constructions that become highly significant at such a time. This information addressed the three elements of duration, commonality and transiency.

Duration. Those with chlamydia frequently ask 'Where did I get it from?' and 'How long have I had it?', reflecting the difficulty in determining the length of an infection that is largely asymptomatic. Such questions are potentially fraught with emotion because they represent a threat to relationships, bringing the sexual behaviour of one's self and one's partner into question. If there is no means by which to accurately determine the length of infection, there is no means by which to attribute its presence to the possibility of a concurrent relationship and the potential threat that this may pose to the relationship. For some respondents the possibility that they had had the infection for a long time was a cause of concern, not least because it may have caused damage to the reproductive system. However, focusing upon the inability to determine how long the infection had been present, and considering the possibility that it had been there for a long time, provided the means by which to understand it as something that was brought into the relationship at the outset rather than introduced at a later stage. For Yvonne, who had been in a stable relationship for 7 years, the possibility that she had had the infection for a long time was important because of the safety that it offered to her relationship.

"We were fine once we'd come here [to the GUM clinic] and they explained that it could lie dormant for quite a few years, it's not forced to be anyone playing away or anything." [Yvonne, 25]

Commonality. When the respondents found that they did have chlamydial infection, the sense of discomfort that it generated generally precluded any wish to discuss the diagnosis with others. There is a high degree of information control and secrecy associated with STIs; chlamydia is predominantly a secret condition. The effect of this is that even though chlamydia is a common infection, it is not very common for someone to know of someone else who has had that infection. This situation creates the illusion that acquiring the infection is a rare occurrence. Consequently, in the absence of any other information, those who found that they had the infection assumed that it was rare. The resultant feeling of isolation further contributed to their sense of dirtiness or otherness.

"Because I think at first, if the doctor says that to you [the diagnosis of infection] and you just feel so dirty as though you're the only one that it's happened to." [Jane, 26]

To discover that this infection was common was crucial in undermining feelings of contamination because it challenged these perceptions. For two of the respondents this was experiential: through the discovery of friends or acquaintances who had also had the infection. Liz described how knowing someone who had had this infection three times had made her feel better, whilst Paula identified how nice it had been to find someone else to talk to who had also been through the experience.

In other cases the relief came simply from being told that these infections were common.

"At first I felt uncomfortable with it, but when they explained it wasn't that serious and lots of people get it, I felt a little bit better about it." [Sarah, 26]

The metaphor of likening chlamydial infection to the common cold was frequently used by many of the staff in the GUM clinic, particularly the health advisor, and was recounted by a number of respondents. The term 'common cold' subtly portrays the significance of this comparison. Although the cold is recognised as a contagious condition passed from one person to another, it is an everyday occurrence, accepted as something to which we are all susceptible and to which we all succumb. Consequently there is no culpability and recrimination associated with its transmission. This understanding serves to challenge the whole social representation of the infection and may therefore reduce the perceived necessity for secrecy and the consequent impression of this infection as rare and therefore isolating.

Transiency. The knowledge that chlamydial infection is extremely responsive to treatment and can be rapidly and effectively eliminated from the body in the vast majority of cases, notwithstanding the possibility of residual pathological damage, played a key role in moderating the emotional responses to diagnosis. Many of the respondents reported a singular lack of understanding of the infection in terms of treatment and management, and they expressed extreme anxiety as to the consequences of the diagnosis. The knowledge that the infection could be eliminated by antibiotic therapy effectively undermined the majority of those initial responses.

"But they said we'll give you some treatment, some tablets, they told me that over the phone, so it like calms your nerves when they say it's just tablets to cure it." [Robert, 19]

"Well, when I knew it were easily treatable, it stopped me worrying about it as much once I did realise that I'd got it.” [Shirley, 16] 


\section{ARTICLE}

The feeling of dirtiness emanated from knowing that they had the infection, that the infective organism was resident within their body. Consequently, elimination of the infection was associated with diminution of the feelings of dirtiness and the expressions of bodily contamination.

\section{Discussion}

These data indicate that the diagnosis of a chlamydial infection is often a cause of considerable distress and upset; however, the way in which the information is conveyed can have a significant impact upon that response, either reinforcing or diminishing the resultant feelings of bodily discomfort and pollution. In this respect the health professional and client interface is a highly sensitive one. The reactions of those health professionals who are involved in all aspects of the infection management come under intense scrutiny because the information that they convey is not biomedically neutral: it is charged with moral significance to the recipient who is highly sensitive to any inference of culpability or social labelling of behaviour at this time.

In relation to felt stigma, expectation of social sanctions from others may cause an individual to overreact in interactions; felt stigma in this respect is characterised by an amplification effect. The way that information is conveyed at all stages, the subtleties of communication and the content of explanations have a considerable effect upon the way that such information is received and the resultant impact upon the individual. Insensitive communications produce bad experiences that appear to have a devastating effect, whilst sensitive communications produce experiences that are positive in their effect, neutralising many of the initial concerns associated with diagnosis.

Melville et al. ${ }^{16}$ propose a model of psychosocial responses to herpes simplex virus within which moderators are those factors that act as buffers to emotional and psychosocial responses. These include the information provided at diagnosis. In this study, discussion of the indeterminacy, the commonness and the treatability of infection serve as moderators because they provide a means by which to facilitate shedding of felt stigma and thereby reduce the overall emotional impact of the infection. These findings demonstrate the importance of careful and sensitive communication that acknowledges and addresses the primary concerns associated with this infection.

There are limitations to the present study. The majority of those interviewed were attending the GUM clinic for follow-up. The relatively high rate of non-attendance associated with this visit indicates that not everyone who has had this infection considers it important to return for follow-up. This necessarily limited the range of experiences reflected in these data and therefore represents a potential source of bias.

Recruitment through a third party was necessary for practical and ethical reasons, however it was problematic at times, being sensitive to organisational and staffing considerations in the clinics, and was largely responsible for poor recruitment at the second site. As a result it was not possible to include the experiences of those who had not attended the GUM clinic.

Males comprised only $20 \%$ of the sample, largely as a result of the recruitment process; fewer were invited to participate, particularly at the beginning of the study, and no males were recruited from the second site. The findings indicate similarities between the males and females, both in terms of their initial responses and the elements that were important in modifying those responses. These findings should, however, be treated with caution because the imbalance limited opportunity for gender comparisons.

Specific methodological considerations in this study include the location of the interviews and the status of the researcher as a health professional, which will have inevitably impacted upon what is voiced within an interview to an indeterminate extent. ${ }^{17}$ Finally, although there was ongoing discussion of the findings, most notably with the research supervisor, the analysis was conducted solely by the researcher, which arguably represents a limitation of interpretation.

\section{Conclusions}

This study illustrates the impact of diagnostic communication upon the way in which people receive and respond to a diagnosis of chlamydial infection. It demonstrates the importance of sensitive management and the provision of contextualised information in undermining the feelings of dirt and contamination that commonly accompany this diagnosis. This information, which represents the patient perspective, is particularly pertinent at this time as the number of health professionals involved in the management of chlamydia increases.

Statements on funding and competing interests

Funding. None identified.

Competing interests. None identified.

References

1 Health Protection Agency (HPA). Diagnoses of Selected STIs by Region, Age and Sex Seen at GUM Clinics: National Level Summary Tables 1995-2003. London, UK: HIV and Sexually Transmitted Infections Department, Health Protection Agency, 2004.

2 Adams EJ, Charlett A, Edmunds RJ, Hughes J. Chlamydia trachomatis in the United Kingdom: a systematic review and analysis of prevalence studies. Sex Transm Dis 2004; 80: 354-362.

3 Underhill G, Hewitt G, McLean L, Randall S, Tobin J, Harinda V. Who has chlamydia? The prevalence of genital tract Chlamydia trachomatis within Portsmouth and South East Hampshire, UK. $J$ Fam Plann Reprod Health Care 2003; 29(1): 17-20.

4 Pimenta JM, Catchpole M, Rogers PA, Perkins E, Jackson N, Carlise C, et al. Opportunistic screening for genital chlamydial infection. I. Acceptability of urine testing in primary and secondary healthcare settings. Sex Transm Infect 2003; 79: 16-21.

5 Faxelid E, Krantz I. Experiences of disease and treatment among chlamydia patients. Scand J Caring Sci 1993; 7: 169-173.

6 Duncan B, Hart G, Scoular A, Biggrig A. Qualitative analysis of psychosocial impact of diagnosis of Chlamydia trachomatis: implications for screening. BMJ 2001; 322: 195-199.

7 Scambler G, Hopkins A. Being epileptic: coming to terms with stigma. Sociol Health Illn 1986; 8: 26-43.

8 Foley E, Patel R. Destigmatising STIs: remaining challenges, new opportunities. Sex Transm Infect 2001; 77: 306-307.

9 Goffman E. Stigma: Notes on the Management of Spoiled Identity. London, UK: Penguin Books, 1990.

10 Scoular A, Duncan B, Hart G. "That sort of place ... where filthy men go ...": a qualitative study of women's perceptions of genitourinary medicine clinics. Sex Transm Dis 2001; 77: 340-343.

11 Pitts MK, Woolliscroft J, Cannon S, Johnson I, Singh G. Factors influencing delay in treatment seeking by first-time attenders at a genitourinary clinic. Int J STD AIDS 2000; 11: 375-378.

12 Gott CM, Rogstad KE, Riley V, Ahmed-Jushuf I. Delay in symptom presentation among a sample of older GUM clinic attenders. Int $J$ STD AIDS 1999; 10: 43-46.

13 Glaser B, Strauss A. The Discovery of Grounded Theory: Strategies for Qualitative Research. New York, NY: Aldine de Gruyter, 1967.

14 Strauss A. Oualitative Analysis for Social Scientists. Cambridge, UK: Cambridge University Press, 1987.

15 Strauss A, Corbin J. Basics of Qualitative Research: Grounded Theory Procedures and Techniques. Newbury Park, CA: Sage, 1990.

16 Melville J, Sniffen S, Crosby R, Salazar L, Whittington W, DithmerSchrek D, et al. Psychosocial impact of serological diagnosis of herpes simplex virus type 2: a qualitative assessment. Sex Transm Infect 2003; 79: 280-285.

17 Miller T. Shifting layers of professional, lay and personal narratives. In: Ribbens J, Edwards R (eds), Feminist Dilemmas in Qualitative Research. London, UK: Sage, 1998; 58-71. 